\title{
Quantum device microfabrication: Resolution limits of Ion beam patterning
}

\author{
A. Scherer and M. L. Roukes \\ Bellcore, Red Bank, New Jersey 07701
}

(Received 6 February 1989; accepted for publication 16 May 1989)

\begin{abstract}
We have achieved precise control of the lateral electrical damage accompanying ion beam patterning of submicron electron devices from semiconductor materials. In situ transport measurements, made in the etching chamber during the definition process, provide an exact and reproiucible means of end-point detection, irrespective of material structure and ion beam parameters. We apply this to routinely fabricate conducting channels having widths below 100 $\mathrm{nm}$ from high-mobility GaAs/A1GaAs heterojunctions. Subsequent low-temperature magnetotransport measurements and successive optimization of processing conditions enable us to systematically obtain the minimum conducting width. Through these studies we explore the ultimate resolution limits of the ion beam patterning process.
\end{abstract}

Current research on the patterning of ultrasmall electronic devices is motivated by the desire to explore and, ultimately, utilize the physics of quantum transport. Advances in both microfabrication and the growth of higin-mosility two-dimensional electron gas (2DEG) heterostructures have recently made it possible to investigate electrical transport in channels where clectrons propagate ballistically, and current is carried by only a few one-dimensional subbands. ${ }^{1,2}$ Fabricating these electron waveguides has been problematic, however, because of the extreme sensitivity of the GaAs to processing induced defects. ${ }^{3}$ The epitaxially grown $2 \mathrm{DEG}$ heterojunctions employed are highly optimized structures. The long scattering lengths (i.e., high mobilities) obtained at low temperatures in material before patterning are easily lost after introduction of only moderate amounts of ior beam damage. ${ }^{4}$ This limitation poses a serious barrier to entering the size regime below the Fermi wavelength, $\lambda_{F}$, where quantum confinenent effects become strongly manifested. In a typical $2 D E G$ material having carrier density $n_{s} \sim 10^{11} \mathrm{~cm}^{-2}, \lambda_{F} \sim 100 \mathrm{~mm}$. In this work the important problem of controlling ion beam induced electrical damage in GaAs is addressed. We focus upon optimizing the fabrication of sub 100 nm quantum wires as a concrete, yet representative, case study.

By using indirect patterning techniques, it is possible to spatially separate the ion-induced electrical damage from the $2 \mathrm{DEG}$ channel. One such technique, shallow etching to pattern only the donor layer, allows structural (mask) widths $w_{\text {str }}$ of conducting channels to be reduced below the $\sim$ l pm threshold. ${ }^{2}$ Previously we reported our development of an even gentler defirition technique, patterning by selective ion damage, ${ }^{5}$ yielding high-mobility conducting channels with structural widhs below $100 \mathrm{~nm}$. Wires $\leqslant 200 \mathrm{~nm}$ created by this method were the first to clearly show quenching of the Hall resistance.' Since resuiting structures have $w_{\mathrm{cl}} \sim w_{\mathrm{str}}$, this technique has enabled us to fabricate complicated patterns with overall dimensions $<0.5 \mu \mathrm{m}$. The careful introduction of ion beam induced damage is the critical step of this technigue; reproducible fabrication of ultrasmall devices hinges upon precise control of this process. We achieve this by monitoring the evolving electrical characteristics of submicron devices in situ during ion bombardment. Magnetotransport data subsequently obtained at low temperatures permit correlation of room-and low-temperature electrical properties. Through succesive generations of samples, we systematically investigate and optimize the relationship between electrical and structural dimensions.

These studies are carried out on $2 \mathrm{DEG}$ heterojunction material with an initial mobility of $6.5 \times 10^{5} \mathrm{~cm}^{2} / \mathrm{V}$ s and a carrier density of $3.3 \times 10^{13} \mathrm{~cm}^{-2}$. Optical lithography defines the $>1 \mu \mathrm{m}$ features for arrays of $0.8 \times 0.9 \mathrm{~mm}$ devices. Each device comprises twelve $100 \mu \mathrm{m}^{2} \mathrm{Au} / \mathrm{N} / \mathrm{i} / \mathrm{Ge}$ onmic contacts, from which $\mathrm{Cr} / \mathrm{Au}$ connecting lead masks radially converge into a $20 \times 40 \mu \mathrm{m}$ field of view for electron beam ithography. In this field, we use conventional $e$-beam and liftofi techniques to pattern $\mathrm{SrF}_{2}$ masis in the form of the 100-800 nm wide quantum wires uttimately desired. These devices, complete up to the critical ion beam exposure step, are mounted and electrically connected to a sample stage subsequently introduced into an ion beam assisted etching (IBAE) chamber via a load lock. Low-level electrical transport measurements are made continuously during ion bombardment by lock-in detection with computerized data acquisition. The fast sampie tumaround time has enabled the broad investigation of parameters essential to find optimal conditions for patterning. Within the IBAE chamber, a Kaufman ion source generates a Ne ion beam with energies ranging from 100 to $500 \mathrm{eV}$ and ion fuxes from 5 to $20 \mu \mathrm{A}$ / $\mathrm{cm}^{2}$. Inert gas flow is regulated at $10 \mathrm{sccm}$, yielding a chamber pressure of $1 \times 10^{-4}$ Torr. We employ Ne as the ion species since the results obtained clearly epitomize the ion damage process. Elsewhere, we describe how resolution limits for ion beam patterning vary with ion species."

Figure 1 displays how the resistance $R$ of three narrow wires having $w_{\mathrm{str}} \sim 100,300$, and $500 \mathrm{~nm}$ evolves with increasing exposure to a $\sim 200 \mathrm{eV}$ Ne beam. Three regions of distinctly different behavior are observed [Fig. 1(a)]. For small ion doses, $\eta<3 \times 10^{16} \mathrm{~cm}^{-2}$, the constant low resistance measured is characteristic of devices before ion bombardment. At the definition dose for these structures, $\eta_{\text {cef }}-3 \times 10^{16} \mathrm{~cm}^{-2}$, unmasked regions of $2 \mathrm{DEG}$ are regdered nonconducting and narrow conducting channels become defined bencath the mask. Here, the mask pattern is transferred into the $2 \mathrm{DEG}$ and the resistance rises abruptly to an initial resistance $R_{i}$ determined by mask geometry and $2 D E G$ sheet resistance $R_{[]}$. Above $\eta_{\text {def }}$ the resistance continues to rise-gradually for the widest wire, and rapidly for the narrowest. Ultimately, $R$ rises steeply as the curoff dose $\eta_{\mathrm{c},}$ 
is approached. In the case of the 300 nm wire this is clearly apparent for $\eta>3 \times 10^{17} \mathrm{~cm}^{-2}$. The evolution of $R$ with $\eta$ bears strong similarity to the $R$ versus gate voltage characteristics for channels defined electrostatically by pinched gates. $^{\text {? }}$

An approximate translation from $R$ to $w_{\mathrm{e} 1}$ can be made using the measured lengths of our devices and $R_{\mathrm{L}, 1}{ }^{8}$ Figure 1 (b) displays $w_{\mathrm{e}}$ vs $\eta$ for the three narrow channels of Fig. 1(a). The electrical width drops rapidly to approach the mask width near $\eta_{\text {cef }}$, then decreases more slowly once the channel is defined. The dose range $\eta_{\text {def }}<\eta<\eta_{\text {co }}$ defines a process window for defining conducting channels-this becomes very narrow for small $w_{\text {str }}$. Note also that $\eta_{\text {der }}$ is the same for all wire widths of Fig. 1(b). If the specimen preparation, sample geometry, ion beam collimation, and the background chamber pressure, among other factors, are not optimal, $\eta_{\text {ief }}$ can become highly irreproducible. For narrow process windows this irreproducibility makes it nearly impossible to deliver the optimal ion dose for patterning. Our in situ end-point detection scheme eliminates these difficulties.

Figure 2(a) displays $R$ ys $\eta$ for a family of $400 \mathrm{~nm}$ wires exposed to Ne ion beams of energies $\sim 130,200,300$, and 500 $\mathrm{eV}$. Figure $2(\mathrm{~b})$ shows $w_{\mathrm{el}}$ vs $\eta$ curves translated as discussed previously. Two trends are immedately apparent: $\eta_{\text {def }}$ decreases and $R_{i}$ increases as the ion energy is increased. The inset of Fig. 2(a) shows, however, that the etch rate increases by only $50 \%$ over the range of ion energies studied, whereas $\eta_{\text {def }}$ increases by almost an order of magnitude. This strong energy dependence is also evident in the inset of Fig. 2(b), where values of both $w_{\mathrm{cl}}$ and the etch depth, just at the point of definition, plotted as functions of ion energy. This clearly shows that the electrical width shrinks rapidly when higher energies are used, whereas the amount of material removed at the definition point actually decreases. We
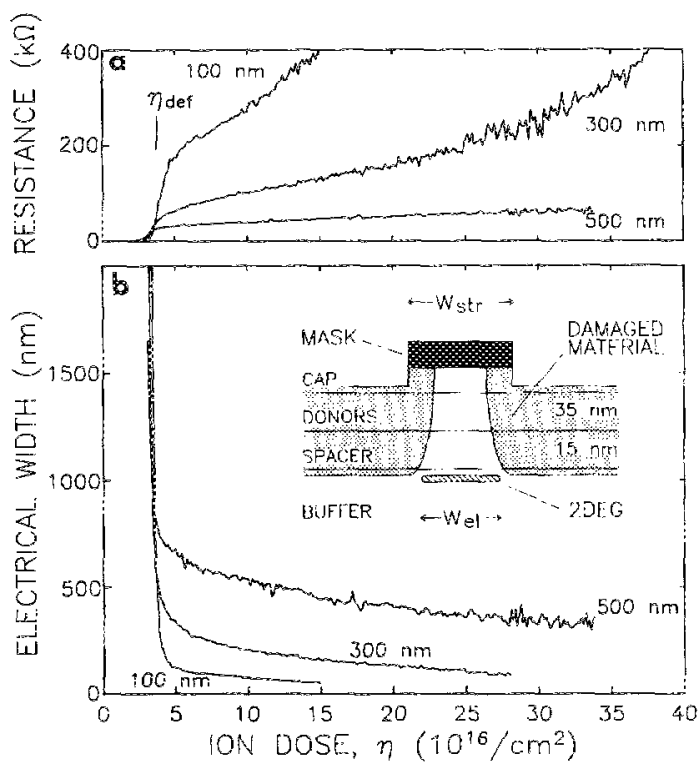

FIG. 1. (a) (Top) Evolution of resisłance with ion dose, measured during bombardment, for $12-i \mathrm{~m}-\mathrm{l}$ ong wires with structural (mask) widths $w_{1,1}$ as shown. At the definition dose, $\eta_{\mathrm{duf}} \sim 4 \times 10^{1 \mathrm{6}} / \mathrm{cm}$, narrow channels are created. (b) (Bottom) Approximate electrical width deduced from the curves of (a). Inset: schematic cross section of a GaAs/AlGaAs $2 \mathrm{DEG}$ wire patterned by selective damage. Only a small amount of material is act aally etched away. picture this behavior as arising from a region of very high resistivity, created by the ion bombardment. The resistivity of this region and its lateral extent grow with both increasing $\eta$ and ion energy. At $\eta_{\text {def }}$, this region spreads laterally under the mask as ion energy increases, resulting in smaller electrical widths at definition for higher energies. This resistance rise can result from increased scattering, carrier depletion, or both, depending on beam parameters and ion species. ${ }^{4,6,9}$ In the case of $\mathrm{Ne}$, it is clear that, despite the shallow etch at the surface achieved at $\eta_{\text {def }}$, the 2 DEG located dcep below is very strongly affected. This clearly points out the important conceptual difference between structural damage and electrical damage-structural damage is local in nature, electrical damage, however, can have substantial remote impact. This is especially true in remotely doped heterostructures such as high-mobility 2DEGs. Recently, Davies has theoretically investigated related problems. ${ }^{10}$

Investigation of few subband transport in narrow wires becomes possible at low temperatures. The electrical characteristics of ion damage-patterned narrow wires change, however, upon cooling from room to low temperatures. To obtain quantitative information, a series of forty-eight $400 \mathrm{~nm}$ wires were fabricated and defined with specific, progressiveiy increasing, ion doses near $\eta_{\mathrm{dcr}}(300 \mathrm{~K})$. At each chosen $\eta$, we simultaneously expose four samples with lengths $l=20$, 40,80 , and $160 \mu \mathrm{m}$. The average resistivities $R / /$ from each set of four, measured at both 300 and $78 \mathrm{~K}$, generate the points plotted semilogarithmically in Fig. 3 . The striking

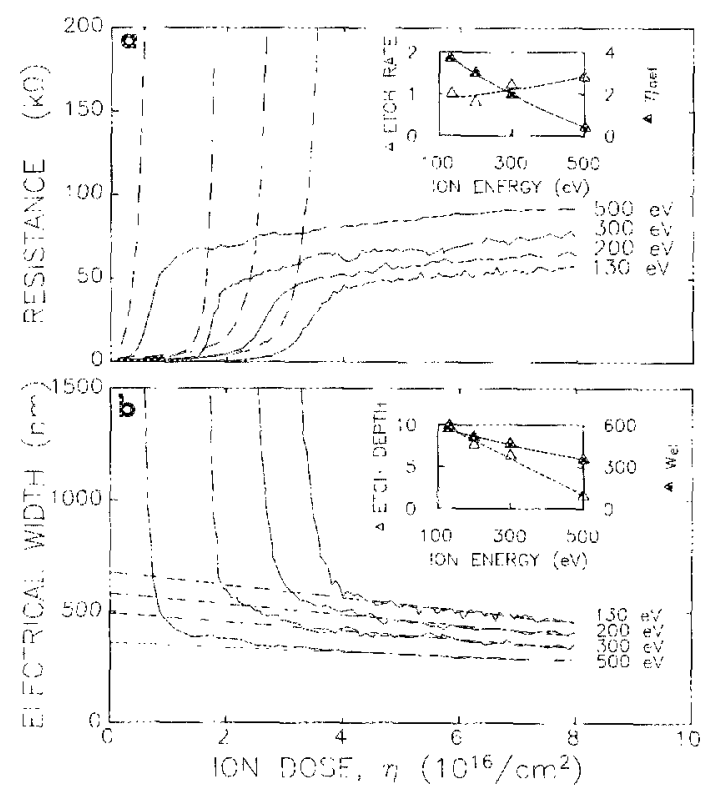

FIG. 2. (a) (Top) Resistance vs ion dose for a serits of $w_{\mathrm{xtr}}=400 \mathrm{~nm}$ wires cxposed at different ion energies. The dashed curves show the behavior of wires patterned to include a small break in the mask. Inset: the definition dose $\eta_{\text {sinf }}$ (in $10^{16} / \mathrm{cm}^{2}$ ) depends strongly on ion energy. The etch rate (in $\mathrm{nm} / \mathrm{min}$ ) obtained using a $10 \mu \mathrm{A} / \mathrm{cm}^{2} \mathrm{Ne}$ flux, however, shows only weak depencience. (b) (Bottom) Approximate electrical widths vs ion dose axtracted from the curves of (a). At $300 \mathrm{~K}$, bow-level residual conduction through unmasked, damaged regions of karge area causes $w_{\mathrm{e}}$ to appear slightly larger than $w_{\text {str }}$ near $\eta_{\text {def }}$. Inset etch depth and electrical widt at the point of definition $\eta_{\text {det }}(E)$, plotted in $1 \mathrm{~m}$, as functions of ion energy $E$. The amount of material physically removed at $\eta_{\text {der }}(E)$ actually decreases at higher energies, while the depletion length $l_{B}(E) \cdots\left[w_{\mathrm{str}}-w_{\mathrm{el}}(E)\right] / 2$ grows dramatically. 


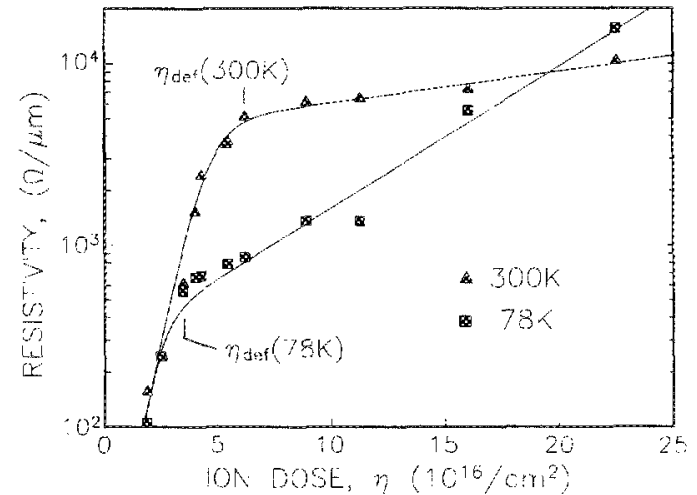

HG. 3. Resistivity $(R / 1) \vee s \eta$ for a series of $w_{\mathrm{st}}=400 \mathrm{~nm}$ wires fabricated using $N_{c}$ at $200 \mathrm{eV}$ and measured at 300 and $78 \mathrm{~K}$. Hack point on the full curve is the inverage of eight measurements on samples of different lengths. A simple expression describing the parallel sam of exponentially increasing resistivities fits the data over several decades of resistance. At $78 \mathrm{~K}$, a high rate of ateral depletion above $\eta_{\text {ctef }}(78 \mathrm{~K})$ is readily apparent.

diference between room-temperature and liquid-nitrogen temperature resistivity curves is apparent: $\eta_{\text {def }}$ moves down by roughly a factor of 2 when the wire is cooled. Wires patterned with doses in the range $\eta_{\mathrm{der}}(78 \mathrm{~K})<\eta<\eta_{\mathrm{def}}(300 \mathrm{~K})$ actually freeze into definition when cooled. Above $\eta_{\text {def }}$ the resistivity rises steeply at low temperatures. This appears to reflect an increasing density of electron traps, created by ion damage, which have activation energies smaller than $300 \mathrm{~K}$. A strong increase in $R$ near $200 \mathrm{~K}$ and much slower cecrease thereafter, for wires given $\eta_{\text {sef }}(78 \mathrm{~K})<\eta<\eta_{\text {def }}(300 \mathrm{~K})$, provides further evidence.

The resolution limit inherent to the ion beam process can be explored once the low temperature $R$ vs $\eta$ characteristic near the point of definition has been obtained. We accomplished this by fabricating three sets of samples, each set comprising 16 wires having widths $w_{\mathrm{str}}=100,200,400$, and $800 \mathrm{~nm}$. All samples within each set are simultaneously exposed using at specific ion dose near the definition threshold. Three doses are chosen, approximately corresponding to $\eta \sim \eta_{\text {def }}(78 \mathrm{~K}), \eta_{\text {der }}(300 \mathrm{~K})$, and $2 \eta_{\text {def }}(300 \mathrm{~K})$. We characterize each Hall geometry sample by magnetotransport measurements at $4 \mathrm{~K}$, performed without illumination, to ascertain that well-formed narrow channels are defined. $R_{x x}$ and $R_{x y}$ traces with negligibie admixture, i.e., flat quantum Hall plateaus at quantized values and strong $R_{x x}$ zeros, indicate that contributions to the conductivity from parallel conduction paths are minimal and electron density is spatially homogeneous. Figure 4 shows $w_{\mathrm{el}}$, normalized to $w_{\mathrm{str}}$, deduced from resistance data taken at $78 \mathrm{~K}$ from these samples. We invoke a simple picture in which a dose-dependent depletion length $l_{D}(\eta)$, acting on each side of the conducting channel, reduces the structural width to the electrical width $w_{\mathrm{el}}=w_{\mathrm{str}}-2 l_{D}(\eta)$. Electrical width shrinks to zero when $w_{\text {str }}$ approaches the cutof width $2 l_{D}$. This model allows us to fit each $w_{\mathrm{el}}\left(\eta, w_{\mathrm{str}}\right)$ measured and, by extrapolation, to obtain an average depletion length for each dose using $2 i_{D}(\eta)=w_{\text {str }}-R_{\square} l / R\left(\eta, w_{\text {sir }}\right)$. Here, $l$ is the channel length. The inset of Fig. 4 displays how the average $l_{D}(\eta)$ increases with $\eta$ beyond $\eta_{\text {def }}(78 \mathrm{~K})$. At the point where $\eta_{\text {def }}(300 \mathrm{~K})$ has been delivered to the sample, $i_{D}$, has

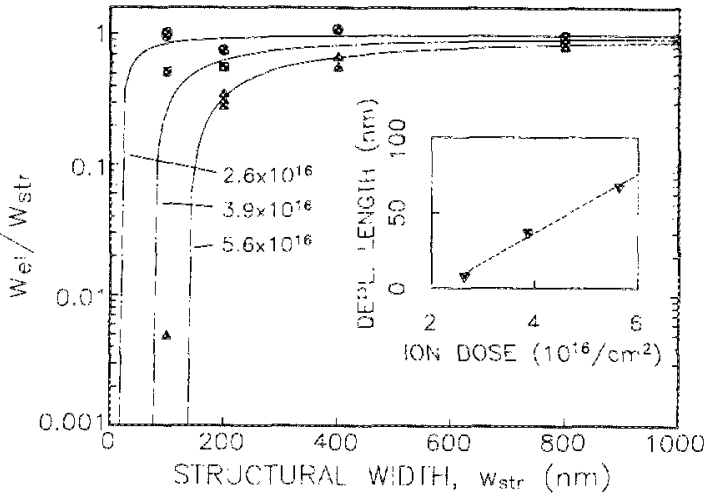

FIG. 4. Electrical width at $78 \mathrm{~K}$ normalized to the structural width for three set of wires exposed to end poirts near $\eta_{\text {def }}(78 \mathrm{~K})$. The solid lines show best fits to the data using a simple depletion model. These vanish at twice the best-fit depletion length $2 l_{D}(\eta)$, where the electrical width shrinks to zero. Inset: the average depletion length rapidly increases beyond its minimum value $l_{D}=15 \mathrm{~nm}$ obtained at the optimal dose $\eta_{\text {ief }}(78 \mathrm{~K})$. Application of ion doses smaller than this value yields wires which are incompleteiy defined at $4 \mathrm{~K}$.

increased by a factor of 5 beyond its optimum value obtained at $\eta_{\mathrm{def}}(78 \mathrm{~K})$. For the particular $2 \mathrm{DEG}$ heterojunction and ion beam conditions employed, we find that a depletion width $l_{D} \sim 15 \mathrm{~nm}$ is obtained at $\eta=\eta_{\text {def }}(78 \mathrm{~K}) \sim 2.5 \times 10^{16}$ $\mathrm{cm}^{-2}$.

In summary, we demonstrate the use of low-energy ion damage, generaily an undesirable result of dry etching, to routinely produce $\sim 100$ nm channels from high-rnobility GaAs/AlGaAs heterojunctions. In sizu electrical measurements provide the precise control of ion beam exposure required and are used to explore the paranetric dependence of the process. We report an optimization procedure which, for the first time, enables conducting widths to be systematically minimized. Through these studies we explore the ultimate resolution limits of ion beam processing.

We gratefully acknowledge the contributions of $B . P$. Van der Gaag, T. L. Cheeks, E. D. Beebe, R. J. Martin, and B.S. D. Lin to this work.

'M. I. Roukes, A. Scherer, S. J. Allen, Jr. H. G. Craighead, R. M. Ruthen, E. D. Beebe, and J. P. Harbison, Phys. Rev. I ett. 59, 3011 (1987).

${ }^{2}$ H. van Houten, B.J. van Wees, M. G. J. Heijmann, and J. P. Andre, Appl. Bhys. Lett. 49,1781 (1986); G. Timp, A.M. Chang, P. Mankiewich, R. Behringer, J. L. Cunninghan, T. Y. Chang, and R. E. Howard, Phys. Rev. l.ett. 5\$. $732(1987)$

${ }^{3}$ S. W. Pang, M. W. Geis, N. N. Efremow, and G. A. Lincoln, J. Vac. Sci. Technol. B 3, 398 (1985); S. K. Ghandi, P. Kwan, K. N. Bhat, and J. M. Borrego, IEEE J. Electron. Device Lett. GDL-3, 48 (1982).

${ }^{4}$ T. L. Cheeks, M. L. Roukes, A. Scherer, B. B. Van der Gaag and H. G. Craighead, in Advances in Materidk, Processing and Devices in III-VCompound Semiconductors, Mater. Res. Soc. Proc, Boston, MA, Fall 1988 (Materials Reasearch Society, Pittsburgh, PA, 1988).

${ }^{5}$ A. Scherer, M. L. Roukes, II. G. Craighead, R. M. Ruthen, E. D. Beebe, and J. P. Hurbison, Appl. Phys. I ett. 51, 2133 (1987)

M. L. Roukes, A. Scherer, and R. P. Van der Gaag, (unpublished).

T. J. Thorton, M. Pepper, H. Ahmed, D. Andrews, and G. J. Davies, Phys. Rev. Lett. 56, 1198 (1986).

${ }^{\text {W }}$ e expect the implicit assumption, that $R_{\mathrm{L}}$, is constant and equal to the broad channel value across $w_{\mathrm{c} 1}$, is ronghly correct so that qualitative trends may be evaluated.

'T. L. Cheeks, M. L. Roukes, A. Scherer, and H. G. Craighead, Appl. Phys. Lett. 53, 1964 (1988).

10J. H. Davies, Semicond. Sci. Technol. 3, 995 (1988). 